$>$ La mort cellulaire joue un rôle fondamental dans le maintien de l'intégrité de l'organisme et son dérèglement a été identifié dans de nombreux processus pathologiques. Une activation en cascade de protéases à cystéine - les caspases - conduit au clivage de divers substrats protéiques essentiels à la survie cellulaire et permet l'amplification d'un signal de mort provenant principalement de deux voies: (1) l'une déclenchée par des protéines relarguées par des mitochondries; (2) l'autre engendrée dans la membrane plasmique par des récepteurs, membres de la famille du TNFR (tumor necrosis factor receptor). Pour chacune de ces voies, les mécanismes d'intégration des signaux s'établissent au niveau d'un complexe multiprotéique, respectivement l'apoptosome et le DISC (death inducing signaling complex). C'est en agissant sur la formation et/ou sur l'activité de ces complexes que la plupart des modulateurs de la mort cellulaire exercent leur action. Par ailleurs, des complexes multiprotéiques localisés dans d'autres lieux stratégiques de la cellule sont également indispensables à l'intégration et/ou à la régulation des signaux de mort cellulaire. <

\section{Régulation de la mort cellulaire programmée : vers une conception plus dynamique}

\author{
Arnaud Couzinet, Zoltán Hérincs, \\ Anne-Odile Hueber
}

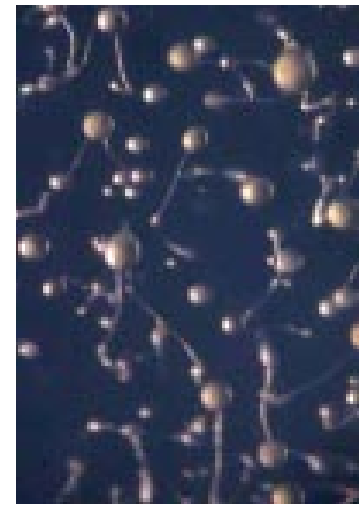

grammées physiologiques est probablement une des meilleures preuves de cet engouement. II a été introduit en 1972 par J.F. Kerr et A.H. Wyllie et désigne une séquence d'altérations morphologiques comprenant une condensation du cytoplasme et du noyau, une fragmentation de l'ADN, un bourgeonnement de la membrane plasmique [2] et une perte de l'asymétrie membranaire. La définition de l'apoptose reposant uniquement sur des critères morphologiques, ce terme est apparu depuis 2 à 3 ans trop restrictif pour définir l'ensemble des morts cellulaires programmées (certaines ne s'accompagnant pas forcément de cette séquence stéréotypée de modifications) [3, 4], et l'on préfère parler maintenant de mort programmée au sens large. Après cette phase purement phénoménologique, les recherches sur la mort cellulaire ont connu une accélération moléculaire, notamment avec le travail pionnier de B. Horvitz sur le développement du nématode C. elegans [5], qui a permis d'identifier l'existence d'un programme moléculaire permettant aux cellules de déclencher leur mort. Au cours du développement de ce ver, 131 cellules sur 1090 meurent au cours d'un processus faisant intervenir quatre protéines-clés, chacune constituant maintenant

Un glossaire regroupe l'ensemble des abréviations utilisées dans les articles traitant de l'apoptose, p. 881. 
le prototype d'une famille de gènes chez les mammifères (Figure 1) : CED-9 (I’homologue des protéines antiapoptotiques de la famille $\mathrm{Bcl}-2$ ) en interagissant avec CED-4 (I'homologue de la protéine pro-apoptotique Apaf-1), l'empêche d'activer CED-3 (I'homologue de la caspase $1 / I C E)$. La mort cellulaire est déclenchée quand la protéine $\varepsilon G L-1$ (I'homologue des protéines pro-apoptotiques de la famille $\mathrm{Bcl}-2$ à domaine $\mathrm{BH} 3$ ) se lie à CED9 et l'inactive, libérant ainsi CED-4 qui peut alors s'oligomériser avec CED-3 et provoquer son auto-activation. Les cellules de mammifères expriment plusieurs membres de chacune de ces quatre familles de protéines et ont ainsi élaboré des réseaux complexes de signalisation conduisant à la mort cellulaire.

\section{Les caspases : acteurs principaux dans l'étape d'exécution}

$(\rightarrow) \mathrm{m} / \mathrm{s}$ $1998, n^{\circ} 1$, p. 9

La première évidence du rôle primordial des protéases à cystéine, appelées plus tard caspases (cysteinyl aspartate-specific proteinases) $(\rightarrow$ ) dans l'étape d'exécution d'un signal de mort provient de l'identification, puis du clonage, du gène pro-apoptotique ced-3 chez $C$. elegans [6], dont le premier homologue identifié chez les mammifères a été ICE (interleukin-1 $\beta$ converting enzyme), plus tard rebaptisé caspase 1 . On connaît maintenant 14 caspases différentes. L'ensemble des membres de cette famille d'endoprotéases possède un site catalytique comprenant un résidu cystéine localisé dans un motif PACXG (où le résidu $X$ est $R, Q$ ou $G$ et le résidu cystéine est directement impliqué dans le processus catalytique). Ces enzymes reconnaissent, puis clivent, des chaînes polypeptidiques au niveau d'un résidu aspartique de la partie carboxy-terminale. La spécificité des caspases pour leur substrat est déterminée en particulier par la nature du résidu en position P4 du site de clivage/reconnaissance (WEXD, DEXD ou [L/V]EXD) qui a permis de définir trois sous-familles. Ces enzymes existent dans le cytoplasme sous une forme inactive (zymogène). Leur protéolyse (par d'autres caspases ou par ellesmêmes grâce à leur activité auto-catalytique) provoque la dimérisation des formes zymogènes conduisant à leur clivage. Un premier clivage libère une première sous-unité ( $\mathrm{pl0/p12),} \mathrm{tandis} \mathrm{qu'un} \mathrm{second} \mathrm{clivage} \mathrm{engendrera} \mathrm{une}$ sous-unité de taille plus importante portant le site catalytique (p17/p20) (Figure 2A). L'enzyme active ainsi formée est composée de deux hétérodimères composés chacun de l'association des deux sous-unités (et comprend donc deux sites catalytiques) (pour revue, voir [7]).

Toutes les caspases possèdent, en position amino-terminale, un pro-domaine dont la taille, variable, est à l'origine d'un mode d'activation particulier qui a permis une classification supplémentaire de ces enzymes (Figure 2B).
- Les caspases à long pro-domaine dites caspases d'amont ou « initiatrices » contiennent des motifs d'interaction protéine-protéine, tels que les domaines DED (death effector domain) pour les caspases 8 et 10 ou CARD (caspase recruitement domain) pour les caspases 1, 2, 4 et 9, par l'intermédiaire desquels elles sont recrutées au niveau de complexes protéiques de signalisation puis auto-activées.

- Les caspases 3, 6, 7 et 14 possèdent quant à elles un pro-domaine plus court (10 à 40 résidus) et sont dites caspases d'aval : ne possédant pas de domaine leur permettant d'être recrutées et de s'oligomériser, elles sont activées à la suite de leur clivage par une autre caspase (à pro-domaine court ou long) ou par la granzyme $B$, aboutissant à un fonctionnement dit en cascade. Leur activation conduit au clivage de diverses protéines substrats (pour revue, voir [8]) à l'origine de la plupart des événements biochimiques, structuraux ou morphologiques de l'apoptose.

\section{La mise en route: \\ quelles sont les voies moléculaires conduisant à l'activation des caspases ?}

L'activation en cascade des caspases permet une amplification d'un signal initial. À ce jour, deux grandes voies moléculaires principales conduisant à l'activation des caspases ont été identifiées: (1) la voie déclenchée

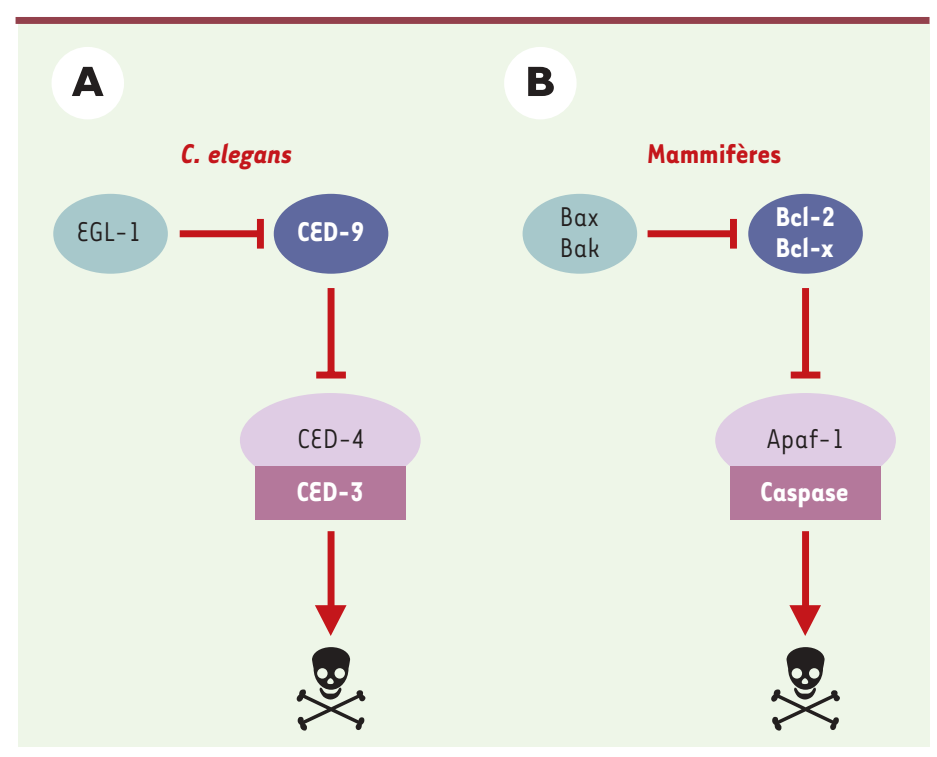

Figure 1. Les protéines essentielles au programme de mort cellulaire chez C. elegans et chez les mammifères. Les études génétiques chez $C$. elegans (A) ont permis de définir quatre protéines-clés essentielles au programme de mort cellulaire chez ce nématode et dont les homologues maintenant identifiés chez les mammifères $(\boldsymbol{B})$ constituent les prototypes de familles de gènes. 
par des protéines relarguées par des mitochondries endommagées; (2) la voie déclenchée au niveau de la membrane plasmique par l'activation de récepteurs membranaires.

\section{La voie intrinsèque mitochondriale}

La mitochondrie, depuis longtemps identifiée comme l'organite indispensable à la production d'ATP, a été plus récemment également impliquée dans la régulation des mécanismes moléculaires de mort cellulaire. En effet, dans une cellule engagée dans un processus de mort cellulaire (à la suite de stimulus engendrant un stress ou un dommage cellulaire, que ce soit à la suite d'un choc thermique ou osmotique, de radiations ionisantes, ou d'un sevrage en cytokines), on observe une perméabilisation des membranes mitochondriales (à l'origine de la libération de protéines pro-apoptotiques de l'espace intermembranaire vers le cytosol) accompagnée dans la plupart des cas d'une chute du potentiel transmembranaire mitochondrial $\left(\Delta \Psi_{\mathrm{m}}\right)$. Le mécanisme précis de cette perméabilisation membranaire reste encore très controversé. Plusieurs modèles sont proposés et pourraient coexister (Figure 3).

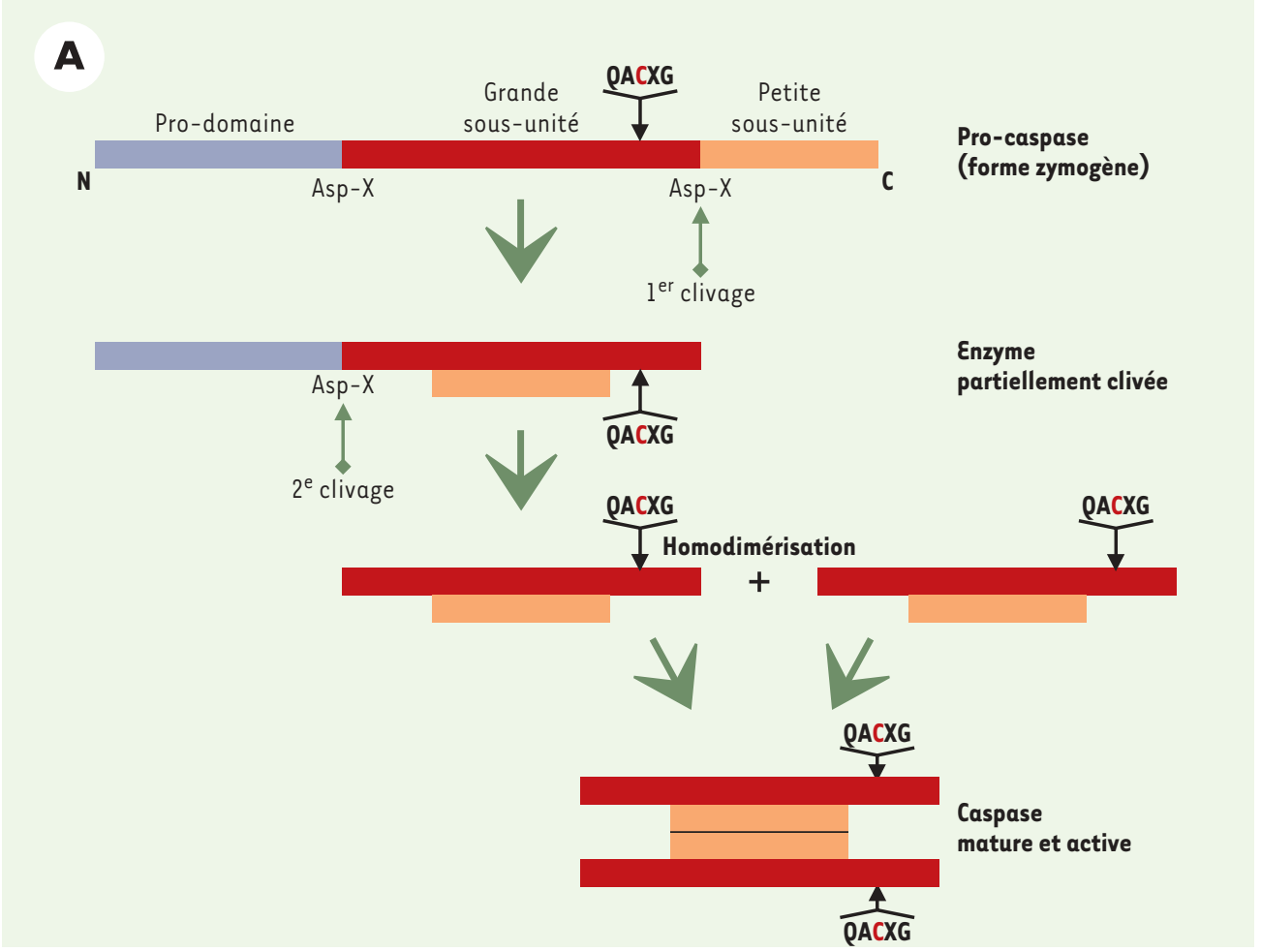

Figure 2A. Activation des caspases. Dans leur forme inactive (zymogène), ces enzymes sont constituées d'un prodomaine, d'une grande sous-unité (portant le site catalytique) et d'une petite sous-unité. Leur clivage en deux étapes successives, libérant d'abord la petite sous-unité puis la grande, conduit à la formation de la forme active de l'enzyme : deux hétérodimères composés chacun de l'association des deux sous-unités, et comprenant donc deux sites catalytiques.
- Une des hypothèses postule l'ouverture d'un mégapore mitochondrial appelé PTPC (permeability transition pore complex) [9], complexe multiprotéique composé en majorité de la porine de la membrane mitochondriale externe (aussi appelé VDAC, voltage dependent anion channel), et d'un «translocateur » de nucléotides à adénine (ANT), inséré dans la membrane mitochondriale interne. Selon ce modèle, des protéines proapoptotiques (telle que Bax), en s'associant au PTPC, favoriseraient son ouverture, provoquant ainsi un gonflement de la matrice mitochondriale (dû à une entrée d'eau et de solutés provoquée par une hyperosmolarité de la matrice). Lors de cette expansion, l'excédent de surface de la membrane interne par rapport à la membrane externe provoquerait une rupture mécanique de cette dernière.

- Un autre modèle suppose un dysfonctionnement de l'échange ATP/ADP mitochondrial lié à la fermeture de VDAC, provoquant une accumulation de protons dans l'espace intermembranaire, responsable à la fois du gonflement de la matrice et d'une production de radicaux oxygénés. Ce modèle ne permet cependant pas d'expliquer comment une libération des protéines apoptogènes pourrait précéder la chute du $\Delta \Psi_{\mathrm{m}}[10]$.

- Un dernier modèle suggère plutôt la formation d'un pore dans la membrane mitochondriale externe permettant la libération de protéines dans le cytosol sans altération physique de la mitochondrie (voir plus loin). La protéine proapoptotique Bax pourrait être à l'origine de la formation de ce pore, soit en interagissant directement avec des lipides, soit en s'associant au VDAC ou bien encore par auto-oligomérisation ou formation d'oligomères avec un autre membre de la même famille [11].

Les protéines ainsi libérées sont maintenant regroupées sous le nom générique de SIMP (soluble inter membrane mitochondrial proteins) et possèdent toutes une 
activité pro-apoptotique (Figure 4). Une première classe de SIMP agit sur la voie classique d'apoptose dépendante des caspases. On y distingue des pro-caspases, le cytochrome $c$ et un répresseur d'inhibiteur de caspases, la protéine Smac/DIABLO. Une fois libéré dans le cytosol, le cytochrome c interagit avec l'homo$(\rightarrow) \mathrm{m} / \mathrm{s} \quad$ logue de CED-4, la protéine Apaf-l et la forme zymo$1996, n^{\circ} 4, \quad$ gène de la caspase 9 , formant ainsi, en présence d'ATP, p. 541 un complexe multiprotéique appelé apoptosome à l'origine du clivage - et donc de la formation de la forme active - de la pro-caspase 9 [12]. Cette dernière activera à son tour d'autres caspases exécutrices comme les caspases 3 ou 7. La protéine Smac/DIABLO, quant à elle, se lie aux protéines inhibitrices de l'apoptose (IAP) et les inactive (voir plus loin).

L'AIF est, pour l'instant, le seul représentant d'une autre classe de SIMP : il s'agit d'une flavoprotéine qui déclenche - d'une manière indépendante des caspases la fragmentation de la chromatine en particules de haut poids moléculaire [13].

\section{La voie extrinsèque des récepteurs membranaires}

Certains des membres de la superfamille des TNFR (tumor necrosis factor receptor) $(\rightarrow)$, connus sous le nom de « récepteurs de mort », ont la particularité de posséder dans leur portion intracellulaire une région conservée appelée le «domaine de mort » (DD, death domain), un motif protéique d'environ 80 acides aminés nécessaire à la transmission du signal de mort par ces récepteurs. La découverte de ces récepteurs de surface induisant spécifiquement un signal de mort a, au cours de ces dernières années, largement contribué à une meilleure compréhension des mécanismes moléculaires mis en place lors de l'induction de la mort cellulaire programmée. Les sous-

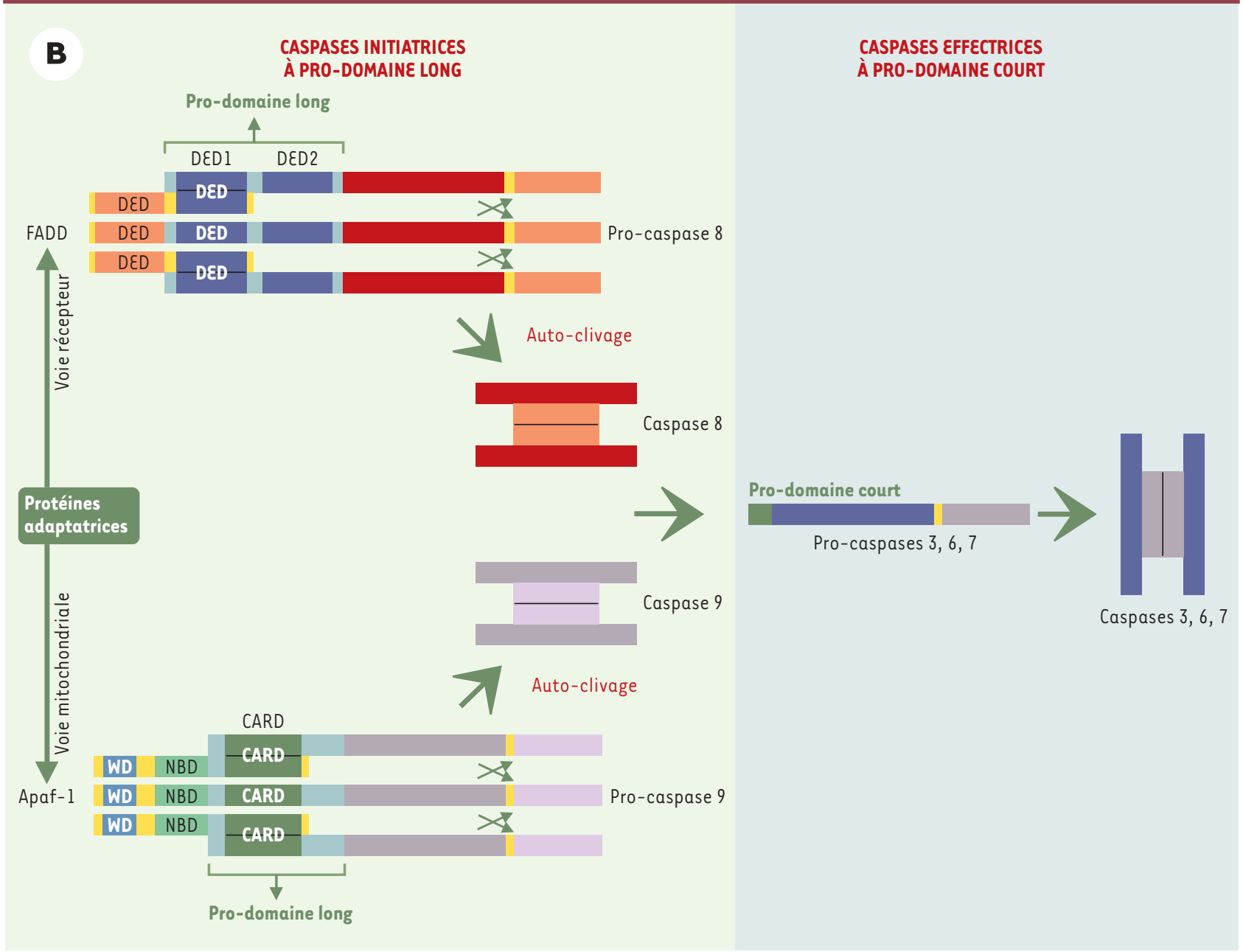

Figure 2B. Exemple d'activation séquentielle des caspases. Les pro-caspases initiatrices possédant un long pro-domaine (pro-caspase 8 ou 9) s'auto-clivent à la suite de leur interaction avec des protéines adaptatrices possédant des motifs identiques (DED ou CARD), puis activent des pro-caspases à pro-domaine court comme la pro-caspase 3. CARD : caspase recruitment domain; DED : death effector domain ; WD : 40 répétitions tryptophane/acide aspartique; NBD : nucleotide binding domain. 
membres de cette famille sont au nombre de 7 : Fas (CD95/AP0-1), TNFRl (p55/CD120a), DR3, DR6, p75NGFR (nerve growth factor receptor), ainsi que les deux récepteurs de TRAIL, les molécules DR4 (TRAIL-R1) et DR5 (TRAIL-R2) (pour revue, voir [14]).

La capacité d'induction de mort cellulaire relayée par le récepteur Fas ayant été la première décrite, nous avons choisi cette voie de signalisation pour illustrer l'ensemble des voies de ces récepteurs de mort [15] $(\rightarrow)$. La voie de signalisation en aval de Fas conduisant à la mort cellulaire est particulièrement bien définie (Figure 4). La recherche des molécules se liant à sa région cytoplasmique a tout d'abord conduit à l'identification de $F A D D$, une protéine cytoplasmique ubiquitaire qui contient, dans sa partie carboxy-terminale, un domaine de mort. L'oligomérisation de Fas permet le recrutement à la membrane de FADD grâce à une interaction homotypique entre domaines de mort. Outre son domaine de mort, FADD possède dans sa partie amino-terminale un

autre domaine appelé domaine effecteur de mort (DED), grâce auquel FADD attire à son tour la pro-caspase 8 (la forme zymogène de la caspase 8 ). Le complexe multiprotéique formé à la membrane par Fas, FADD et la caspase 8 , à la suite de la multimérisation du récepteur Fas par son ligand (Fas-L) a été appelé le DISC (death-inducing signaling complex) [16]. Le rapprochement des pro-caspases 8 dans le DISC permet leur clivage réciproque et la libération des formes actives de la caspase 8 dans le cytosol, qui vont à leur tour cliver un certain nombre de caspases effectrices telles que les caspases 3,6 et 7 .

Jusqu'à présent, il avait été supposé que le ligand de Fas

$(\rightarrow) \mathrm{m} / \mathrm{s}$

1999, n०6-7, p. 909

$(\rightarrow) \mathrm{m} / \mathrm{s}$ 2001, n ${ }^{\circ} 10$, p. 1088 sous forme homotrimérique (composé de trois monomères identiques) engageait trois monomères de Fas conduisant ainsi à l'assemblage d'un récepteur trimérique et à l'agrégation des domaines de mort sous la membrane [17]. Mais ce modèle d'assemblage du récepteur Fas a été récemment remis en cause par les travaux des équipes de M. Lenardo [18] et G. Ruberti [19] qui ont démontré non seulement que Fas possédait la capacité de s'assembler en trimères

A

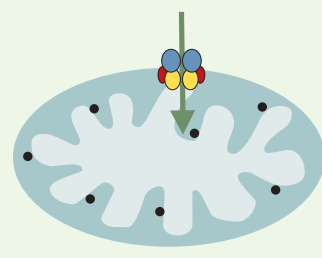

OUVERTURE DE PTPC

B

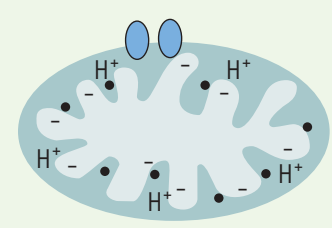

FERMETURE DE VDAC

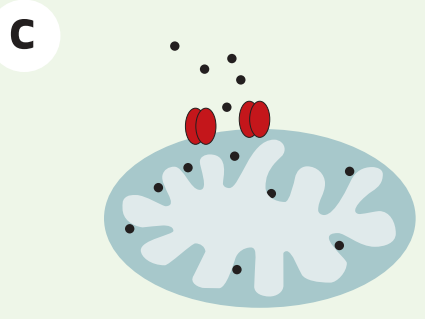

PORES LIPIDIPUES

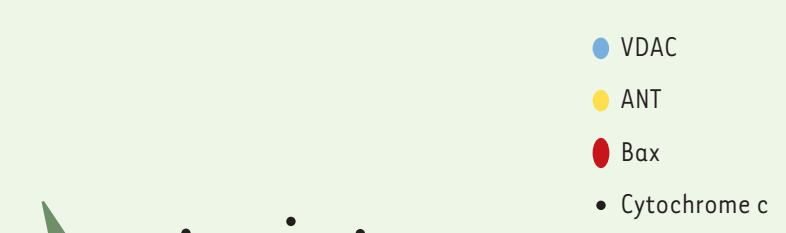

2
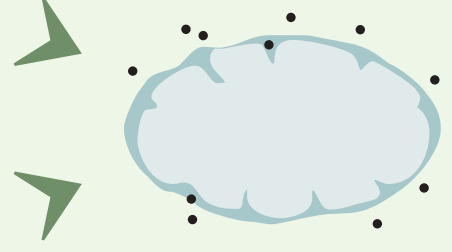

.

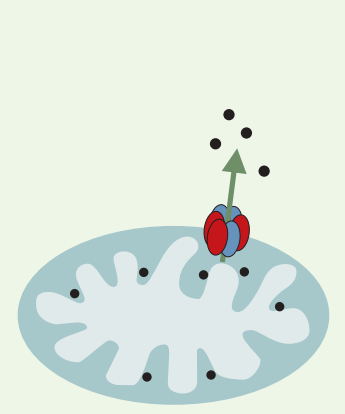

CANAL Bax/VDAC

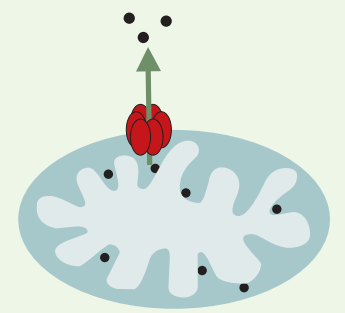

CANAL Bax/Bax
Figure 3. Modèles possibles de libération des SIMP (soluble inter membrane mtichondrial proteins) de l'espace intermembranaire vers le cytosol. Les modèles $A$ et $B$ proposent une rupture mécanique de la membrane externe mitochondriale provoquée par le gonflement de la matrice intermembranaire mitochondriale. Ce dernier serait dû à l'ouverture du PTPC (pore transition permeability complex) (A) ou à la fermeture de la porine (VDAC) (B), entraînant une altération dans l'échange ATP/ADP. Le modèle (C) propose la participation du membre pro-apototique de la famille Bcl-2, Bax, soit dans une régulation des pores lipidiques ou du canal VDAC, soit en formant lui-même un canal à la suite de son oligomérisation. indépendamment de l'engagement de son ligand, mais que ce pré-assemblage des monomères en trimères était un prérequis à la liaison de Fas- $\mathrm{L}$ et donc à la transmission du signal de mort. La région nécessaire à cette trimérisation de Fas appelée PLAD (preligand assembly domain) est constituée des 49 premiers acides aminés de la région amino-terminale de la portion extracellulaire de Fas et est donc distincte du domaine de liaison à Fas-L. Ainsi, indépendamment de la présence d'un ligand, des trimères et des monomères de Fas co-existent dans la membrane plasmique $(\rightarrow)$. Cette découverte soulève évidemment plusieurs questions intéressantes. Ces trimères peuvent-ils engendrer un signal indépendamment du ligand? Dans l'affirmative, est-ce un signal de 


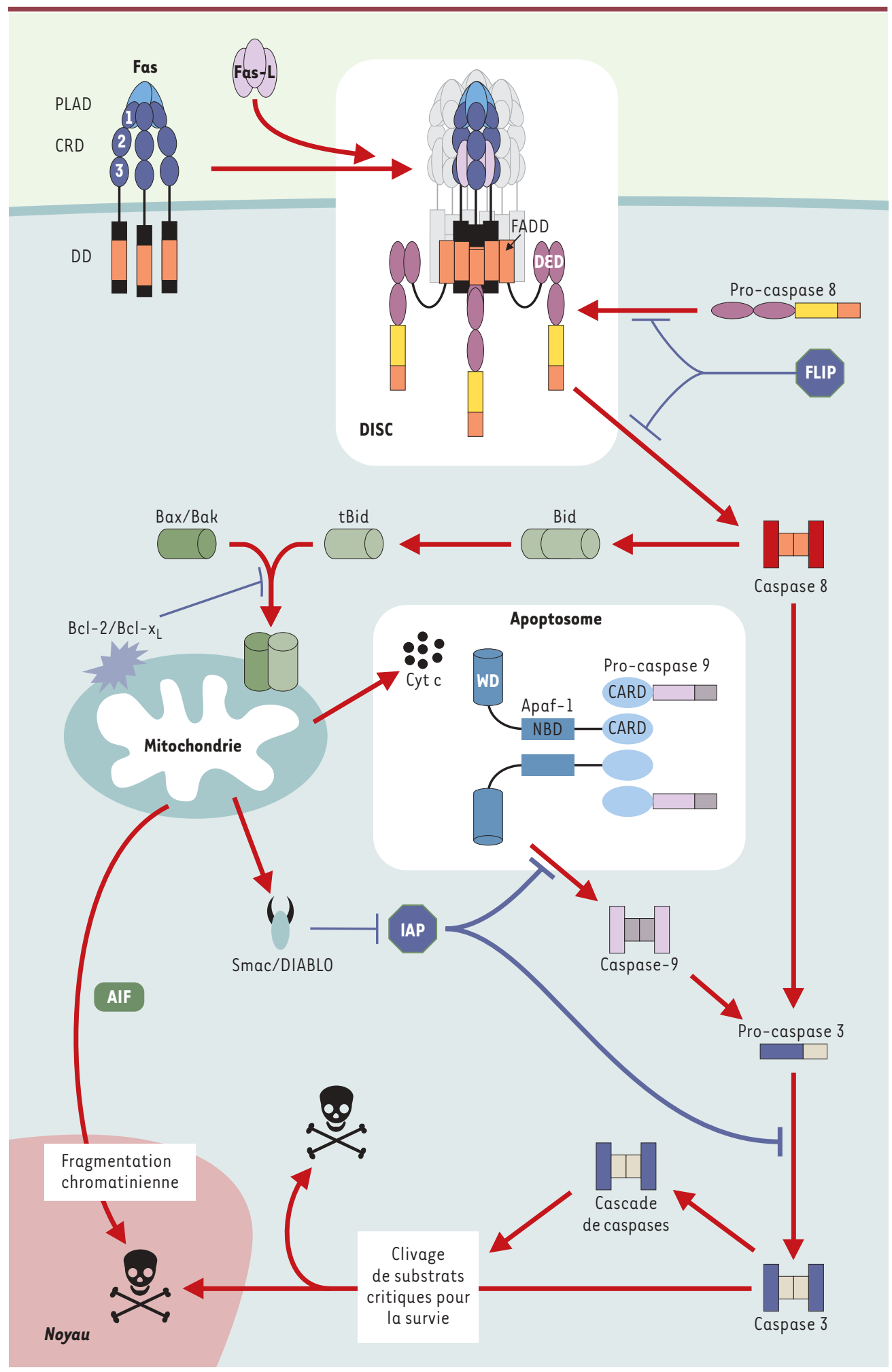

Figure 4. Schéma général réprésentant les deux voies principales de déclenchement de la mort cellulaire. L'oligomérisation de Fas, engendrée par interaction avec son ligand, permet le recrutement de FADD et de la procaspase 8 à la membrane plasmique grâce à des interactions homotypiques entre respectivement les domaines DD (death domain) et DED (death effector domain). Le complexe multiprotéique ainsi formé a été appelé DISC (death-inducing signaling complex). Le rapprochement des pro-caspases 8 dans le DISC permet leur clivage réciproque et la libération de la caspase 8 dans le cytosol, qui va à son tour cliver un certain nombre de caspases effectrices telle que la caspase 3. Une deuxième voie, déclenchée par le clivage de la molécule Bid par la caspase 8 , est caractérisée par une perméabilisation des membranes mitochondriales

aboutissant au relargage dans le cytosol des SIMP (le cytochrome c, Smac/DIABLO et AIF) : le cytochrome c interagit avec la protéine Apaf-1 (apoptotic protease activating factor) permettant, en présence d'ATP, le recrutement de la pro-caspase 9, formant ainsi un complexe multiprotéique appelé apoptosome, à l'origine de l'activation de la caspase 9, qui activera à son tour la caspase 3. La protéine Smac/DIABLO, quant à elle, se lie aux protéines inhibitrices de l'apoptose (IAP) et les inactive. Enfin, AIF migre dans le noyau, où elle met en route la fragmentation chromatinienne. WD : 40 répétitions tryptophane/acide aspartique ; NBD : nucleotide binding domain; FLIP : FADD-like ICE inhibitory proteins. 
mort ? L'agrégation de leur domaine de mort suffit-elle à recruter des molécules comme FADD?

\section{Un dialogue étroit entre la voie du récepteur et} la voie mitochondriale

L'identification de la caspase 8 dans le DISC lie directement le récepteur Fas à la cascade des caspases. Son activation via FADD est essentielle à la mort engendrée par Fas : les thymocytes et les fibroblastes embryonnaires de souris déficientes en FADD ou en caspase 8 résistent à la mort induite par Fas. Cependant, l'importance de la voie mitochondriale a été récemment démontrée dans la mort induite par Fas au moins dans certaines cellules [20]. En fait, selon l'utilisation de ces deux voies apoptotiques en aval de Fas, deux types de cellules ont pu être définies [20] : alors que les cellules de type I seraient indépendantes d'une activité apoptotique des mitochondries et utiliseraient uniquement une activité directe des caspases, les cellules de type II en seraient dépendantes. Mise à part la quantité de pro-caspase 8 recrutée et clivée au niveau du DISC (faible dans les cellules de type II et importante dans les cellules de type I), aucune différence moléculaire n'a permis à ce jour d'expliquer cette différence de comportement entre les deux types de cellules.

Un membre de la famille $\mathrm{Bcl}-2$ à domaine $\mathrm{BH} 3$ récemment identifié, la molécule Bid [21], constitue un des liens entre la voie du récepteur et la voie mitochondriale (Figure 4). Un fragment de cette molécule engendré à la suite du clivage par la caspase 8 est transféré du cytoplasme à la mitochondrie. En effet, Bid, en se liant à Bax, un autre membre pro-apoptotique de la famille $\mathrm{Bcl}-2$ présent sous forme monomérique dans le cytosol, induit l'oligomérisation de ce dernier et son intégration dans la membrane externe mitochondriale, entraînant l'ouverture de mégapores mitochondriaux à l'origine de la chute du potentiel transmembranaire mitochondrial et de la libération du cytochrome c [22].

Une autre protéine faisant la jonction entre les deux voies a été identifiée. II s'agit de la protéine régulatrice BAR [23], qui possède la capacité de s'associer à la fois aux molécules anti-apoptotiques $\mathrm{Bcl}-2 / \mathrm{Bcl}-\mathrm{x}_{\mathrm{L}}$ via un domaine SAM et à la caspase 8 via un domaine DED.

Très longtemps, une incertitude a plané sur le rôle inhibiteur de Bcl-2 dans la mort induite par Fas. Une explication possible est venue de l'identification des cellules de type I ou de type II. En effet, $\mathrm{Bcl}-2$ ne jouerait aucun rôle dans la mort provoquée par Fas dans les cellules au sein desquelles une activation directe des caspases est suffisante (type I). En revanche, dans des cellules dans lesquelles une amplification du signal d'activation via la voie mitochondriale (type II) est nécessaire, la mort induite par Fas serait modulée par des protéines de la famille $\mathrm{Bcl}-2$.

\section{Intégration et régulation des signaux au niveau de complexes multiprotéiques}

Pour chacune des deux voies moléculaires décrites (la voie mitochondriale et la voie du récepteur) conduisant à la mort de la cellule, les mécanismes d'intégration des signaux s'établissent au niveau d'un complexe multiprotéique (respectivement l'apoptosome et le DISC). C'est en agissant au niveau de la formation et/ou de l'activité de ces derniers que la plupart des modulateurs de la mort cellulaire exercent leur action.

Les régulateurs

du complexe multiprotéique « récepteur 》

\section{Les récepteurs leurres de Fas}

La plupart des récepteurs du TNF à domaine de mort existent aussi sous une forme soluble (engendrée par épissage alternatif ou par clivage protéolytique à la membrane plasmique); celles-ci bloquent la fonction du récepteur et peuvent même être utilisées comme agent thérapeutique [24]. Un épissage alternatif de I'ARNm de Fas conduit à la production de toute une série de protéines tronquées parmi lesquelles on trouve des formes solubles du récepteur Fas, capables in vitro d'entrer en compétition avec Fas et agissant donc comme des formes dominantes négatives [19, 25]. De façon intéressante, on peut noter que l'ensemble de ces variants possède la région PLAD, responsable de l'oligomérisation des monomères de Fas [19]. Leur capacité d'interférer avec le signal de mort de Fas est donc liée à leur interaction homotypique avec le récepteur Fas, perturbant ainsi son homo-oligomérisation et donc la formation du DISC.

Un gène appelé $D c R 3$ codant pour un récepteur leurre de Fas, membre de la famille TNFR et se liant à Fas-L, a été isolé [26]. Il s'agit d'une protéine sans région transmembranaire et sécrétée. Bien que son homologie avec la molécule Fas ne soit pas très forte, sa capacité de liaison à Fas-L est comparable à celle de Fas. La protéine DcR3 est capable d'inhiber la mort engendrée par Fas, probablement en entrant en compétition avec la molécule Fas au niveau de sa liaison à Fas-L.

\section{La localisation de Fas à la membrane plasmique}

La localisation spécifique, la concentration locale des récepteurs à la membrane, la présence de molécules adaptatrices dans la région sous-membranaire, représentent également des paramètres importants de modulation d'un signal. Dans ce contexte, nous avons récemment mis en évidence le rôle crucial de microdo- 
maines membranaires particuliers riches en sphingolipides et en cholestérol, les rafts (radeaux), dans les signaux de mort cellulaire engendrés par Fas [27]. Nous $(\rightarrow) \mathrm{m} / \mathrm{s}$ 1996, nº 11 , p. 1263 avons montré, d'une part, que les molécules Fas étaient localisées de façon constitutive dans les rafts et, d'autre part, que les molécules FADD et caspase 8 y étaient recrutées après engagement de Fas. Ces régions membranaires apparaissent essentielles à la signalistion de ce récepteur puisque leur absence inhibe non $(\rightarrow) \mathrm{m} / \mathrm{s}$ 1999, n5, p. 745 seulement la formation du DISC à la membrane mais également le signal de mort engendré par Fas. II est donc possible que les rafts puissent servir de platesformes à partir desquelles la cascade de signalisation engendrée par l'engagement du récepteur Fas à la membrane plasmique et conduisant à la mort cellulaire est déclenchée et organisée.

\section{Un récepteur leurre de FADD : FLIP}

Plusieurs régulateurs agissent aussi au niveau de la formation du DISC. Les plus connus sont les FLIP (FADD-like ICE inhibitory proteins), des isoformes de caspase 8 , possédant deux domaines DED mais pas de site catalytique actif (Figure 5A) $(\rightarrow)$. Ces protéines fonctionnent donc comme des molécules leurres de FADD en entrant en compétition avec les caspases 8 pour leur recrutement au niveau du DISC [28]. Bien qu'il existe différents variants transcriptionnels de FLIP, seuls deux sont présents au niveau protéique: une forme longue $c-F L I P L$ et une forme courte $c-F L I P_{s}$. Bien que toutes deux soient capables de bloquer un signal de mort induit par le récepteur Fas, elles sembleraient agir à des niveaux différents du clivage de la pro-caspase 8 [29].

Il est par ailleurs raisonnable d'imaginer que la présence d'autres molécules capables d'interagir avec la région cytoplasmique du récepteur pourraient moduler la liaison (domaine transmembranaire). de protéines adaptatrices au domaine de mort, et donc l'activité du DISC. Leur présence pourrait, entre autres, empêcher une auto-agrégation des récepteurs pouvant engendrer une mort spontanée indépendante d'un ligand. C'est le cas notamment de la molécule SODD (silencer of death domains), qui interagit avec TNFRI [30] $(\rightarrow)$.

\section{Les régulateurs}

du complexe multiprotéique «mitochondrial 》

Les membres

\section{de la famille Bcl-2}

Les membres de la famille $\mathrm{Bcl}-2$ ont une importance cruciale dans la régulation des voies de signalisation de la mort cellulaire. Cette famille comprend aussi bien des protéines pro-apoptotiques (comme Bax, Bak, Bad, Bid, Bim, etc.) qu'anti-apoptotiques (comme Bcl-2 et $\left.B c l-x_{L}\right)$. L'alignement de leur séquence protéique a permis de définir quatre régions de forte conservation

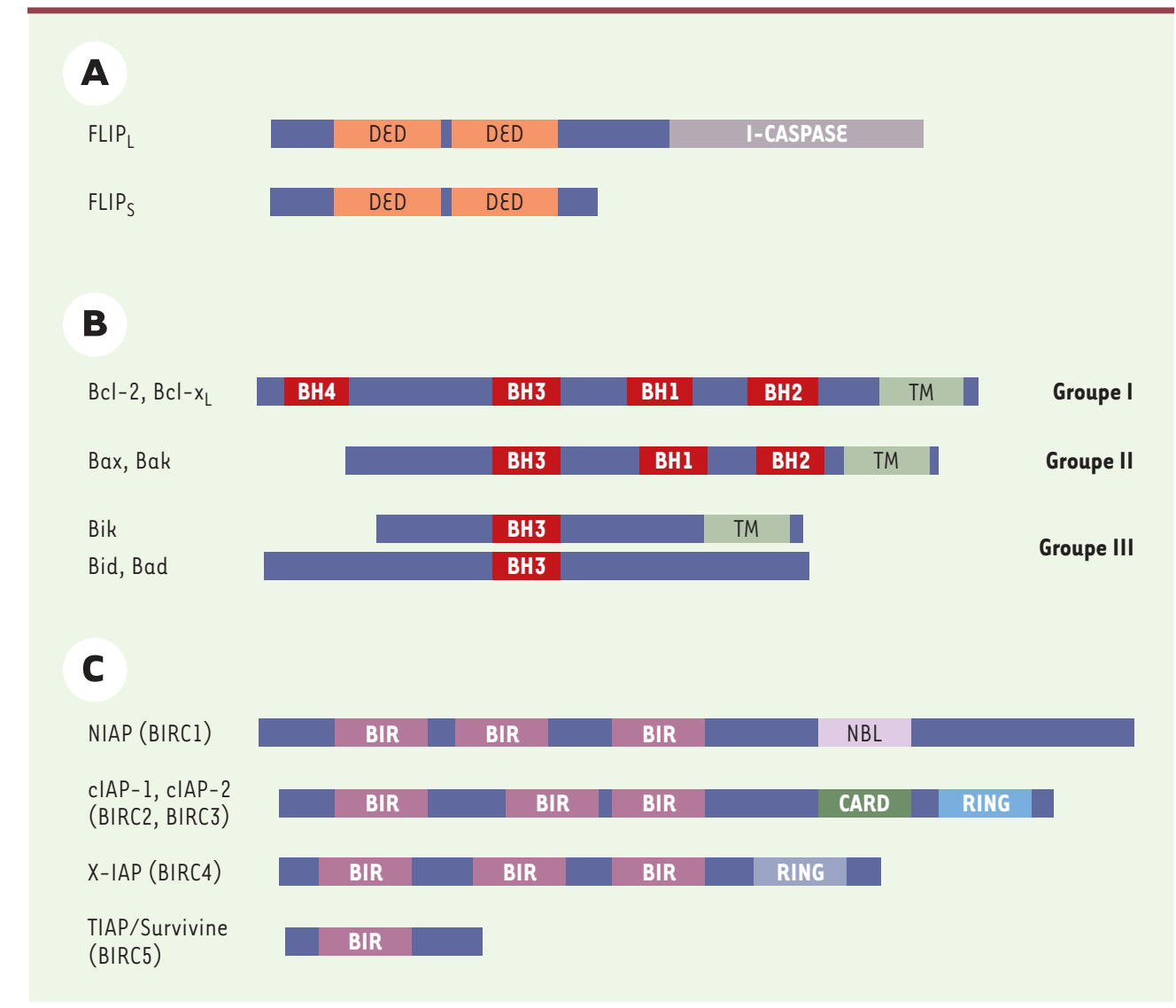

Figure 5. Trois classes principales de régulateurs de la mort cellulaire. FLIP (FADD-like ICE inhibitory proteins) (A), les membres pro- ou anti-apoptotiques de la famille Bcl-2 (B), et les IAP (inhibitor of apoptosis proteins) (C). Les domaines conservés entre les différents membres d'une même famille sont représentés: BH ( $B c l-2$ homology), BIR (baculoviral IAP repeat), CARD (caspase recruitment domain), DED (death effector domain), RING (zinc finger), TM 
appelées domaines $\mathrm{BH}$ ( $\mathrm{BCl}-2$ homology) 1 à 4 (Figure $5 B$ ). Certaines molécules pro-apoptotiques contiennent les domaines $\mathrm{BH} 1, \mathrm{BH} 2$ et $\mathrm{BH} 3$ ( $\mathrm{Bak}$ ), tandis que d'autres ne contiennent qu'un seul domaine BH3 (Bid, Bim, Bad). Le domaine BH4 est, quant à lui, spécifique des protéines anti-apoptotiques $\left(\mathrm{Bcl}-2, \mathrm{Bcl}-\mathrm{x}_{\mathrm{L}}, \mathrm{Bcl}-\mathrm{w}\right.$, MCl-I, Al, Diva). Ces protéines interagissent entre elles formant des homodimères ou des hétérodimères, le niveau relatif de chaque protéine dans la cellule déterminant sa sensibilité à un signal de mort [31]. Par ailleurs, une grande majorité d'entre elles contiennent une partie carboxy-terminale hydrophobe qui assure leur ancrage au niveau de la membrane externe mitochondriale, mais également des membranes externes du réticulum endoplasmique et du noyau.

Les molécules anti-apoptotiques siégeraient au niveau de la mitochondrie tandis que les protéines pro-apoptotiques posséderaient une localisation différente (cytosol ou microtubules). À la suite d'un signal apototique, ces dernières s'inséreraient dans la membrane mitochondriale et induiraient sa perméabilisation [32]. II a été proposé que cette capacité d'insertion soit liée à leur similitude de structure avec certaines toxines bactériennes, leur permettant de former des canaux/pores transmembranaires modulant ainsi la libération des SIMP [33]. D'autres auteurs proposent qu'elles pourraient aussi être liées à leur interaction directe avec VDAC (voir plus haut) [10].

\section{Deux régulateurs principaux des caspases : les IAP et Smac/DIABLO}

Contrairement à d'autres modifications post-traductionelles, le clivage protéolytique des caspases semble irréversible et est donc soumis à un contrôle très strict. Les IAP (inhibitor of apoptosis protein) sont des protéines qui inhibent la mort cellulaire en se liant directement aux caspases (aussi bien initiatrices qu'effectrices), empêchant ainsi leur clivage et leur activité [34]. Toutes possèdent un ou plusieurs motifs BIR (baculoviral IAP repeat) qui pemettent leur interaction avec les caspases et sont donc indispensables à leur activité anti-apoptotique. Certaines contiennent un domaine RING (zinc finger) (Figure 5C). C'est le cas du prototype de cette famille, la molécule XIAP, qui se lie à la caspase initiatrice 9 et aux caspases effectrices 3 et 7, inhibant ainsi leur activité. À son tour, l'activité inhibitrice de XIAP sur les caspases peut être réglée de manière négative par au moins deux protéines interagissant directement avec XIAP : la protéine Smac/DIA$B L O$, une des SIMP, qui, sous sa forme active, se lie à XIAP et inhibe sa capacité de liaison aux caspases, et XAF-1 (XIAP associated factor 1) [35].
Dialogue inter-organites : vers une conception dynamique de la mort cellulaire

Il est probable que d'autres complexes multiprotéiques indispensables à l'intégration et/ou à la régulation des signaux de mort cellulaire existent dans d'autres lieux stratégiques de la cellule. En effet, le rôle de plus en plus précis joué par des organites aussi divers que le réticulum endoplasmique, l'appareil de Golgi ou les lysosomes a été récemment mis en évidence dans la littérature (pour revue, voir [36]). Parmi ces organites se trouve le noyau, dont le rôle a été très étudié et discuté. Il avait d'abord été suggéré (à partir de la présence de modifications morphologiques caractéristiques de l'apoptose dans des cellules enuclées [37]) que la présence du noyau n'était pas requise pour l'exécution de l'apoptose, et que celui-ci ne faisait que subir les événements mis en œuvre pour éliminer la cellule eucaryote. Des découvertes plus récentes suggèrent néanmoins qu'il jouerait un rôle essentiel dans une régulation du programme apoptotique. Nous l'illustrerons ici en commentant trois exemples que nous estimons représentatifs (Figure 6).

1. Les POD (PML oncogenic domain) sont des amas moléculaires sphériques qui, bien qu'exclusivement nucléaires, participent activement au déroulement de la mort cellulaire induite par une variété de stimulus. Le rôle des POD dans la mort cellulaire est illustré par les situations au cours desquelles ils sont absents des cellules. En effet, chez des souris déficientes en PML (promyelocytic leukemia) - protéine indispensable à leur formation - les cellules sont protégées de la mort induite par Fas, le TNF, le céramide, l'interféron, ou l'irradiation $\gamma$ [38]. Certaines pistes moléculaires à la base de ce dialogue entre les récepteurs de mort à la surface cellulaire et ces protéines localisées dans ces structures particulières du noyau, commencent à être bien documentées. C'est le cas de la molécule Daxx (Fas death domain-associated protein) dont la capacité de potentialiser la mort induite par surexpression de Fas dans les cellules Hela a permis de définir un nouvel axe dans la mort induite par Fas [39, 40]. Daxx, bien qu'initialement identifiée comme une molécule se liant au DD de Fas et activant la voie JNK (C-Jun N-terminal kinase) via le recrutement de ASKl (apoptosis signal-regulating kinase 1) [39, 40], a été par la suite décrite comme une protéine nucléaire, capable d'interagir avec PML au niveau des POD (lorsque PML est liée de façon covalente à SUMO-1) et de réprimer la transcription $(\rightarrow)$ [41]. De manière intéressante, la présence de Daxx dans les POD est un pré-requis à son activité pro-apopto- $\rightarrow$ m/s

2000, n' 11 , p. 1242 
tique dans la mort induite par Fas [42, 43]. Cette molécule semble donc être une molécule des POD capable de répondre à un signal envoyé par un récepteur membranaire tel que Fas. Les travaux de l'équipe de J. Landry ont d'ailleurs montré une relocalisation de Daxx dans le cytoplasme à la suite d'une stimulation par Fas [44]. Une autre molécule capable de s'associer à Fas, la sérine thréonine kinase FIST (Fas-interacting serine/threonine kinase), est également présente dans les POD [45]. Cette protéine est capable d'interagir avec FADD, formant un complexe trimoléculaire FasFIST-FADD dans lequel FIST phosphoryle FADD. Par ailleurs, FIST interagit fortement avec Daxx, mais le compartiment cellulaire dans lequel cette interaction a lieu demeure inconnu. II semblerait donc qu'un certain nombre de protéines associées aux POD ne soient pas confinées à ces structures ou présentes exclusivement dans le noyau comme on aurait pu l'imaginer, mais soient capables d'interagir avec des récepteurs membranaires. La résultante est un trafic protéique entre le noyau et le cytoplasme fournissant une source d'échange apparemment indispensable à l'exécution de l'apoptose.

Ces échanges protéiques entre le noyau et le cytoplasme à l'origine d'une régulation de la mort cellulaire ne semblent pas être limités aux seules protéines des POD, comme le démontre le rôle de XAF- 1 et de DEDD.

2. La molécule XAFl est une protéine nucléaire à domaine RING (zinc finger) qui interagit directement avec XIAP, provoquant sa redistribution du cytosol vers le noyau. Ainsi, son expression antagonise l'activité anti-apoptotique de XIAP et augmente le seuil de sensibilité d'une cellule à un signal de mort. Contrairement à l'autre inhibiteur connu de $X I A P$, la molécule Smac/DIABLO, XAFl ne nécessite pas de signal d'activation mais interagit constitutivedu signal apoptotique (voir glossaire, p. 881). ment avec XIAP pour inhiber sa fonction dans des cellules destinées à mourir. Dans cette optique, le noyau est utilisé comme un outil de séquestration de molécules anti-apoptotiques. De façon intéressante, le niveau d'expression de XAFl est très faible dans certaines lignées cancéreuses [46].

3. La molécule DEDD (DED-containing DNA-binding protein) est une protéine localisée majoritairement dans le noyau et se liant à I'ADN $[47,48]$. Elle exerce une activité pro-apoptotique probablement via sa capacité d'activer la caspase 6 et d'inhiber la transcription. Sa rétention dans le noyau et donc son rôle de régulateur apoptotique semblent être modulés par son interaction avec la protéine DEDAF (DED associated factor), très récemment isolée [49]. II est intéresssant de remarquer que, comme Daxx et FIST, DEDAF possède la particularité d'avoir une double localisation. En effet, outre le fait de moduler l'activité de DEDD au niveau nucléaire, elle a été trouvée dans le cytoplasme, favorisant la formation du DISC en interagissant avec les DED de FADD et des pro-caspases 8 et 10 .

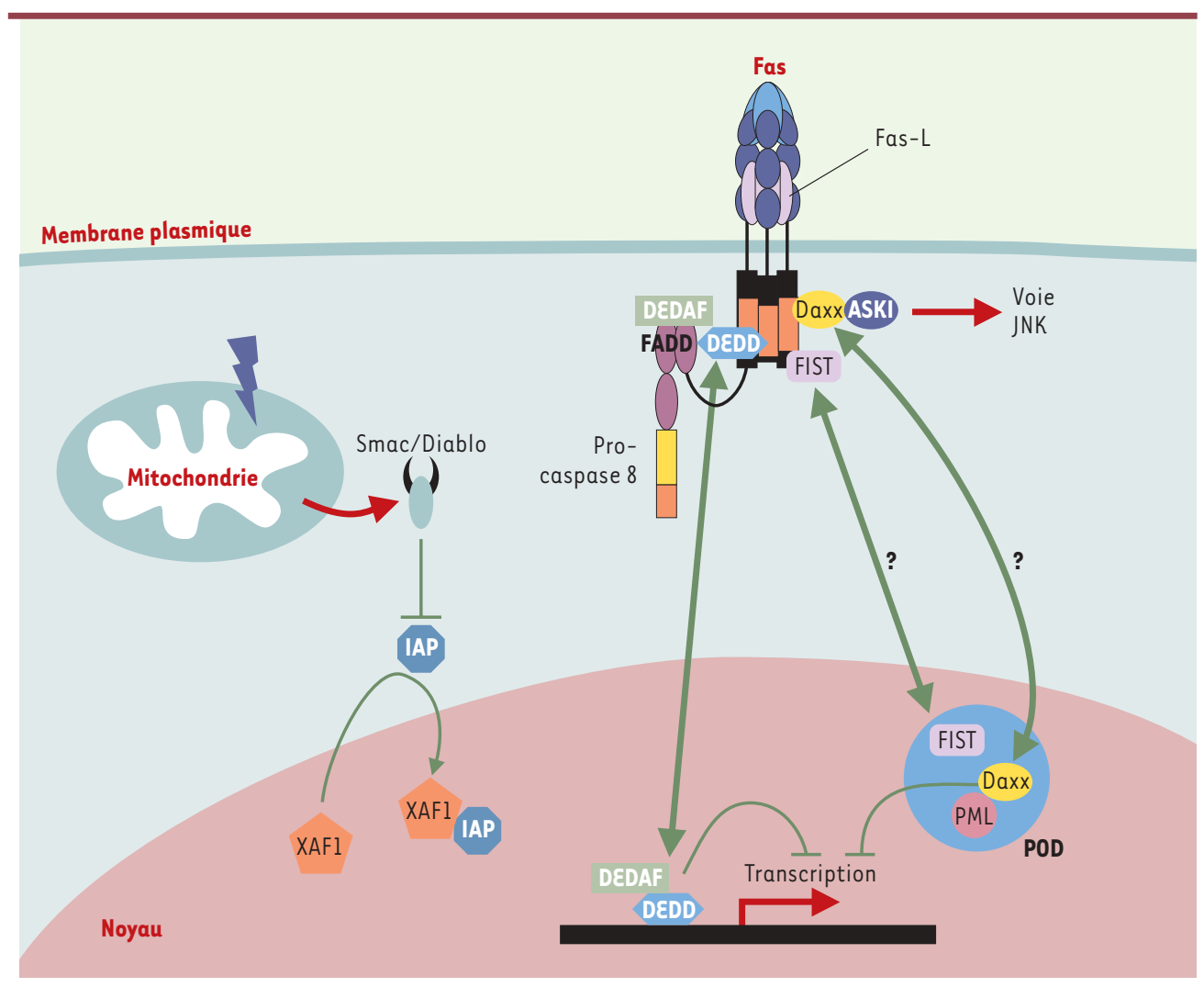

Figure 6. Le dialogue cytoplasme-noyau. Un nombre croissant de protéines réglant la mort de la cellule et possédant la particularité d'avoir une double localisation (cytoplasmique et nucléaire) ont été décrites. Leur relocalisation d'un compartiment cellulaire à l'autre lors d'un stimulus de mort pourrait être un moyen de régulation 


\section{Conclusions}

Au cours de ces dernières années, des analyses biochimiques et génétiques ont donc permis : (1) de définir un grand nombre de voies de signalisation responsables de la mort cellulaire ; et (2) d'identifier leurs principaux acteurs. En dépit de ces avancées théoriques importantes, peu de progrès ont été réalisés dans le traitement ou la prévention des maladies associées au dérèglement de la mort cellulaire (comme les cancers, les syndromes neurodégénératifs et auto-immuns ou les infections virales). Il est raisonnable d'espérer que le développement d'agents pharmacologiques, modulateurs de la mort cellulaire utilisables en thérapie, devrait constituer un des enjeux majeurs de la médecine du XXI ${ }^{e}$ siècle. $\diamond$

\section{REMERCIEMENTS}

Les auteurs remercient vivement les Dr P. Golstein et H.T. He pour la lecture critique de ce manuscrit. Les travaux provenant de notre équipe sont soutenus par le Cnrs (équipe ATIPE), l'Association pour la Recherche contre le Cancer (ARC), la Ligue Nationale pour la Recherche contre le Cancer (LNCC), et la Fondation pour la Recherche Médicale (FRM).

\section{SUMMARY}

Programmed cell death' regulation: Towards a more dynamic conception

Programmed cell death is essential for the development and maintenance of multicellular organisms and alterations in control of cell death/survival contribute to the pathogenesis of many human diseases. Cell death is ultimately executed by caspases, a family of cysteinil aspartate specific proteinases that cleave critical intracellular proteins and execute the apoptotic program. At least two major pathways for caspase activation have been identified: (1) the receptor-mediated pathway which involves members of the tumor necrosis factor (TNF) family of death receptors and (2) the mitochondrial-mediated pathway involving SIMP (soluble inter membrane mitochondrial proteins) released from the mitochondria. A formation of a multi protein complex which forms a template for efficient caspase processing is characteristic for each pathway respectively: the DISC (death inducing signaling complex) is formed by the death receptor and a set of cytosolic adaptor proteins (including procaspase) rapidly recruited to the membrane after ligand binding, and the apoptosome contains cytochrome c, Apaf-l and procaspase 9 . The function of these two complexes is modified at different levels by multiple inhibitory proteins: Flips (FADD-like ICE inhibitory proteins), IAPs (inhibitor of apoptosis protein) and the $\mathrm{Bcl}-2$ family members. The role of other protein complexes formed at other locations within the cell, such as the nucleus that might have a role in cell death regulation will be also discussed. $\diamond$

\section{RÉFÉRENCES}

1. Vogt C. Untersuchungen über die

Entwicklungsgeschichte der Geburtshelferkröte (Alytes obstreticans). Solothurn 1842

2. Kerr JF, Wyllie AH, Currie AR. Apoptosis : a basic biological phenomenon with wide-ranging implications in tissue kinetics. Br J Cancer $1972 ; 26: 239-57$.

3. Wyllie AH, Golstein P. More than one way to go. Proc Natl Acad Sci USA 2001 ; $98: 11-3$.

4. Leist M, Jaattela M. Four deaths and a funeral: from caspases to alternative mechanisms. Nat Rev Mol Cell Biol 2001 ; 2 : 589-98.

5. Horvitz HR. Genetic control of programmed cell death in the nematode Caenorhabditis elegans. Cancer Res 1999 ; 59 (suppl) : 1701-6.

6. Yuan J, Shaham S, Ledoux S, Ellis $\mathrm{H}$, Horvitz $\mathrm{H}$. The $\mathrm{C}$. elegans cell-death gene ced- 3 encodes a protein similar to mammalian interleukin-1 beta converting enzyme. Cell 1993 ; 75 : 641-52.

7. Budihardjo I, Oliver $H$, Lutter $M$, Luo X, Wang X. Biochemical pathways of caspase activation during apoptosis. Annu Rev Cell Biol 1999 ; 15 : 269-90.

8. Thornberry NA, Lazebnik Y. Caspases : enemies within. Science 1998 ; 281 : 1312-6.

9. Marzo I, Brenner C, Zamzami $N$, et al. The permeability transition pore complex: a target for apoptosis regulation by caspases and bcl-2-related proteins. J Exp Med 1998; 187 : 1261-71.

10. Zamzami N, Kroemer G. The mitochondrion in apoptosis: how Pandora's box opens. Nat Rev Mol Cell Biol 2001 ; $2: 67-71$.

11. Martinou JC, Desagher S, Antonsson B. Cytochrome $C$ release from mitochondria: all or nothing. Nat Cell Biol 2000 ; 2 : ع4l-3.

12. Zou H, Li Y, Liu X, Wang X. An
APAF-1 cytochrome $c$ multimeric complex is a functional apoptosome that activates procaspase-9. J Biol Chem 1999 ; 274 : 11549-56.

13. Susin SA, Lorenzo HK, Zamzami N, et al. Molecular characterization of mitochondrial apoptosisinducing factor. Nature 1999 ; 397 : 441-6.

14. Locksley RM, Killeen N, Lenardo MJ. The TNF and TNF receptor superfamilies: integrating mammalian biology. Cell 2001 ; 104 : 487-501.

15. Siegel RM, Chan FK, Chun HJ, Lenardo MJ. The multifaceted role of Fas signaling in immune cell homeostasis and autoimmunity. Nat Immunol $2000 ; 1: 469-74$.

16. Kischkel FC, Hellbardt $S$, Behrmann I, et al. Cytotoxicity-dependent APO-1 (Fas/CD95)associated proteins form a death-inducing signaling complex (DISC) with the receptor. EMBO J 1995 ; 14 : 5579-88.

17. Orlinick JR, Vaishnaw A, Elkon KB, Chao MV. Requirement of cysteinerich repeats of the Fas receptor for binding by the Fas ligand. J Biol Chem 1997 ; 272 : 28889-94.

18. Siegel RM, Frederiksen JK, Zacharias DA, et al. Fas preassociation required for apoptosis signaling and dominant inhibition by pathogenic mutations. Science 2000 ; 288 : 2354-7.

19. Papoff G, Hausler P, Eramo $A$, et al. Identification and characterization of a ligand-independent oligomerization domain in the extracellular region of the CD95 death receptor. J Biol Chem 1999 ; 274 : 38241-50.

20. Scaffidi C, Fulda $S$, Srinivasan A, et al. Two CD95 (APO-1/Fas) signaling pathways. EMBO J $1998 ; 17$ : 1675-87. 
21. Luo X, Budihardjo I, Zou H, Slaughter C, Wang X. Bid, a $B c \mid 2$ interacting protein, mediates cytochrome $\mathrm{c}$ release from mitochondria in response to activation of cell surface death receptors. Cell 1998; 94 : 481-90.

22. Eskes $R$, Desagher $S$, Antonsson B, Martinou JC. Bid induces the oligomerization and insertion of Bax into the outer mitochondrial membrane. Mol Cell Biol $2000 ; 20: 929-35$.

23. Zhang $H, X u Q$, Krajewski S, et al. BAR: an apoptosis regulator at the intersection of caspases and $\mathrm{Bcl}-2$ family proteins. Proc Natl Acad Sci USA $2000 ; 97:$ 2597-602.

24. Feldman $M$, Taylor $P$, Paleolog $\varepsilon$, Brennan FM, Maini RN. Anti-TNF alpha therapy is useful in rheumatoid arthritis and Crohn's disease: analysis of the mechanism of action predicts utility in other diseases. Transplant Proc $1998 ; 30: 4126-7$.

25. Jenkins $M$, Keir M, McCune JM. A membrane-bound Fas decoy receptor expressed by human thymocytes. J Biol Chem 2000 ; 275 : 7988-93.

26. Pitti RM, Marsters SA, Lawrence DA, et al. Genomic amplification of a decoy receptor for Fas ligand in lung and colon cancer. Nature 1998 ; 396 : 699-703.

27. Hueber AO, Bernard AM, Hérincs Z, Couzinet A, He HT. An essential role of membrane rafts in the initiation of Fas/CD95triggered cell death in mouse thymocytes. EMBO Rep $2002 ; 3: 190-6$.

28. Irmler M, Thome M, Hahne $M$, et al. Inhibition of death receptor signals by cellular FLIP. Nature 1997 ; 388 : 190-5.

29. Krueger A, Schmitz I, Baumann S, Krammer PH, Kirchhoff S. Cellular FLICEinhibitory protein splice variants inhibit different steps of caspase- 8 activation at the CD95 death-inducing signaling complex. J Biol Chem 2001; 276 : 20633-40.

30. Jiang $Y$, Woronicz JD, Liu W, Goeddel DV. Prevention of constitutive TNF receptor 1 signaling by silencer of death domains. Science 1999 ; 283 : 543-6.

31. Adams JM, Cory S. The Bcl-2 protein family: arbiters of cell survival. Science 1998 ; $281: 1322-6$.

32. Crompton M. Bax, Bid and the permeabilization of the mitochondrial outer membrane in apoptosis. Curr Opin Cell Biol 2000 ; $12:$ 414-9.

33. Schendel SL, Montal M, Reed JC. Bcl-2 family proteins as ion-channels. Cell Death Differ 1998 ; 5 : 372-80.

34. Fesik SW, Shi Y. Structural biology. Controlling the caspases. Science 2001 ; $294: 1477-8$.

35. Holcik M, Gibson H, Korneluk RG. XIAP: apoptotic brake and promising therapeutic target. Apoptosis $2001 ; 6$ : 253-61.

36. Ferri KF, Kroemer $\mathrm{G}$. Organelle-specific initiation of cell death pathways. Nat Cell Biol 2001 ; 3 : ع255-63.

37. Jacobson MD, Burne JF, Raff MC. Programmed cell death and $\mathrm{Bcl}-2$ protection in the absence of a nucleus. EMBO J $1994 ; 13: 1899-910$.

38. Wang ZG, Delva L, Gaboli M, et al. Role of PML in cell growth and the retinoic acid pathway. Science 1998 ; 279: 1547-51.

39. Yang X, Khosravi-Far R, Chang H, Baltimore D. Daxx, a novel Fas-binding protein that activates JNK and apoptosis. Cell 1997 ; 89 : 1067-76.

40. Chang HY, Nishitoh $H$, Yang $X$, Ichijo H, Baltimore D. Activation of apoptosis signal-regulating kinase 1 (ASKI) by the adapter protein Daxx. Science 1998 ; $281: 1860-3$.

41. Li H, Leo C, Zhu J, et al. Sequestration and inhibition of Daxxmediated transcriptional repression by PML. Mol Cell Biol 2000 ; 20 : 1784-96.

42. Torii S, Egan DA, Evans RA, Reed JC. Human Daxx regulates Fas-induced apoptosis from nuclear PML oncogenic domains (PODs). EMBO J 1999 ; 18 : 6037-49.

43. Zhong $S$, Salomoni $P$, Ronchetti S, Guo A, Ruggero D, Pandolfi PP. Promyelocytic leukemia protein (PML) and Daxx participate in a novel nuclear pathway for apoptosis. J Exp Med 2000 ; 191: 631-40.

44. Charette SJ, Lavoie JN, Lambert $H$, Landry J. Inhibition of Daxxmediated apoptosis by heat shock protein 27. Mol Cell Biol 2000 ; 20 : 7602-12.

45. Rochat-Steiner V, Becker K, Micheau 0, Schneider P, Burns K, Tschopp J. FIST/HIPK3: a Fas/FADDinteracting serine/threonine kinase that induces FADD phosphorylation and inhibits Fas-mediated Jun $\mathrm{NH}(2)$-terminal kinase activation. J Exp Med 2000 ; 192 : 1165-74.

46. Liston P, Fong WG, Kelly NL, et al. Identification of XAFl as an antagonist of XIAP anti-caspase activity. Nat Cell Biol 2001 ; 3 : 128-33.

47. Stegh $A H$, Schickling 0 , Ehret $A$, et al. DEDD, a novel death effector domaincontaining protein, targeted to the nucleolus. EMBO J 1998 ; 17 : 5974-86.

48. Schickling 0 , Stegh $A H$, Byrd J, Peter ME. Nuclear localization of DEDD leads to caspase- 6 activation through its death effector domain and inhibition of RNA polymerase I dependent transcription. Cell Death Differ 2001 ; 8 : 1157-68.

49. Zheng L, Schickling 0 , Peter ME, Lenardo MJ. The death effector domain-associated factor plays distinct regulatory roles in the nucleus and cytoplasm. J Biol Chem 2001 ; 276 : 31945-52.
TIRÉS À PART

A.0. Hueber 